\title{
Ironing it out
}

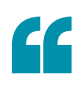

a link between

the activity

of the lipid

peroxidase

pathway and

the high-

mesenchymal

state of

therapy-

resistant

tumours

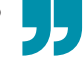

Cancer cells with mesenchymal-like properties are more likely to become resistant to a wide range of therapies. A new study suggests that this mesenchymal-like state of cancer cells is vulnerable to inhibition of the lipid peroxidase pathway, leading to an iron-dependent form of cell death, ferroptosis.

Viswanathan et al. analysed a panel of 516 epithelium-derived cancer cell lines, for which gene expression data sets as well as small-molecule sensitivity measurements for a broad panel of compounds were publically available. Using previously published epithelial-to-mesenchymal transition (EMT) gene signatures, they first computed a mesenchymal score for each cell line. Those were then correlated to small-molecule sensitivity measurements in order to identify potential vulnerabilities.

Two groups of compounds selectively induced death in cells with high mesenchymal scores: a group of agents that induce ferroptosis, a non-apoptotic form of cell death that occurs in response to the accumulation of lipid peroxides, and statins, which target cholesterol synthesis. Through further correlation analysis, inhibition of the enzyme glutathione peroxidase 4 (GPX4) emerged as a key candidate for the mechanism of action of these compounds. The function of GPX4 is to neutralize lipid peroxides that accumulate in the cell and would otherwise cause ferroptosis. Indeed, ferroptosis-inducing agents inhibited
GPX4 activity, and statins reduced its expression, so the researchers studied GPX4 further. In a panel of 610 cancer cell lines of highmesenchymal state, the sensitivity to GPX4 inhibition strongly correlated with the presence of the EMT regulator and lipogenic factor zinc finger E-box-binding homeobox 1 (ZEB1). In KP4 high-mesenchymal state pancreatic cells, ZEB1 deletion reduced sensitivity to GPX4, indicating a functional link. In addition, therapy-resistant cell lines of high-mesenchymal state, such as gefitinib-resistant, mesenchymal-like non-small-cell lung cancer cells, or cells from patients wih BRAF-mutant melanoma, in which BRAF-inhibitor resistance could be induced by transforming growth factor- $\beta$ (TGF $\beta$ ) treatment, also showed dependency on GPX4. This was confirmed in vivo, where knockout of GPX4 in a xenograft model of the therapyresistant, high-mesenchymal state melanoma cell line LOXIMVI led to tumour regression. Finally, when lipid peroxide-producing enzymes upstream of GPX4 were inhibited or reduced, GPX4 inhibition no longer induced cell death in vitro.

Thus, these results provide a link between the activity of the lipid peroxidase pathway and the high-mesenchymal state of therapyresistant tumours, which is bridged by ZEB1, and which could be exploited for the treatment of these tumours.

$$
\begin{array}{r}
\text { Ulrike Harjes, Associate Editor, } \\
\text { Nature Reviews Cancer } \\
\text { This article is modified from the original in Nat. Rev. } \\
\text { Cancer (http://dx.doi.org/10.1038/nrc.2017.71) }
\end{array}
$$

ORIGINAL ARTICLE Viswanathan, V. S. et al. Dependency of a therapy-resistant state of cancer cells on a lipid peroxidase pathway. Nature 547 , 453-457 (2017) 